\title{
Evaluation of chest computed tomography features in COVID-19: a single-center retrospective study
}

\author{
Akshat Sanjay Shukla ${ }^{1 *}$, Sanjay Rajendraprasad Shukla ${ }^{1}$, Feral Ravi Daruwala ${ }^{1}$
}

\begin{abstract}
Background: Even though Real-Time Polymerase Chain Reaction (RT-PCR) is a gold standard for confirming COVID-19, it continues to be plagued by a lack of RT-PCR kits and the potential of false-negative results. Hence, during the second wave of COVID-19 in India, Computed Tomography (CT) scan is an emerging diagnostic tool in evaluating the severity of illness in COVID-19 pneumonia. The present study endeavored to assess chest CT features of COVID-19 pneumonia in Indian population.
\end{abstract}

Methods: This was a single-center, retrospective, observational study conducted in 300 consecutive adults RT-PCR confirmed COVID-19 patients from 1, Jan 2021 to 31, March 2021 at a private radio diagnostic center. Data regarding baseline demographics, clinical and laboratory characteristics, extent, pattern, and type of abnormal CT findings were noted.

Results: The study population (204 males and 108 females) had mean age of $43.18 \pm 8.27$ years. Our study's most common clinical presentation was cough (48.1\%) and fever (47.1\%), respectively. Lung parenchymal abnormalities were found in $294(94.2 \%)$ patients. Abnormal CT findings revealed the involvement of bilateral (45.6\%) and multilobar (42.9\%) with a predominant peripheral (92.3\%) and posterior (80.8\%) distribution. According to the type of opacity, Ground Glass Opacity (GGO) was the dominant abnormality found in 270 (91.8\%) patients, in which pure GGO (36.7\%), GGO with crazy paving pattern (39.8\%), and GGO mixed with consolidation (52.0\%) were noted. Perilesional or intralesional segmental or subsegmental pulmonary vessel enlargement was found in $192(65.3 \%)$ patients.

Conclusion: During the second wave of COVID-19, a chest CT scan is a modality of choice in diagnosing COVID-19 pneumonia and related lung parenchymal changes.

Keywords: COVID-19, Computed Tomography, Lung Disease, Reverse Transcriptase Polymerase Chain Reaction, SARS-Cov-2, India

\section{Background}

In late December 2019, a novel enveloped single-stranded RNA virus, known as Severe Acute Respiratory Syndrome CoronaVirus 2 (SARS-CoV-2), caused a highly infectious disease called CoronaVirus Disease (COVID-19) in Wuhan City, Hubei province, China [1]. The second wave of COVID19 continues to increase its effect worldwide [2] and more so in India. The COVID-19 has been diagnosed in more than 156 million patients worldwide, and more than 3 million infected people have died, whereas, especially in India, more than 21 million confirmed patients with more than 2 lakh deaths have been reported [3]. Clinical presentation of COVID-19 modifies from asymptomatic through symptomatic to critically ill

*Correspondence: akshat.shukla006@gmail.com

${ }^{1}$ Shukla Diagnostic Center, Balaghat, Madhya Pradesh, India

A full list of author information is available at the end of the article patients [4, 5]. Timely and accurate detection of COVID-19 patients are essential to seclude afflicted patients from not infected individuals and restrain its spread. Real-Time Reverse Transcription Polymerase Chain Reaction (RT-PCR) is a standard laboratory technique used for the diagnosis of COVID19 infection; nevertheless, the sensitivity of this technique varies from $42-71 \%$ due to its associated limitations, as such, suboptimal sampling technique, low viral load in the nasopharyngeal region, or impediments in assay performance [6-9]. Importantly, in the second wave of COVID-19, the number of false-negative RT-PCR results has increased dramatically due to a virus mutation; the virus in the current wave of infection has a shorter incubation period and a higher tendency to involve the lungs rather than the upper respiratory tract during incubation period [10, 11]. However, recent studies have revealed the significance of chest Computed Tomography 
(CT) in the detection and diagnosis of COVID-19; chest CT is an excellent diagnostic tool to confirm the lung pathology in suspected COVID-19 patients due to its higher sensitivity (97\%) as opposed to RT-PCR [12-14]. Interestingly, chest CT features of COVID-19 are closely similar to other viral pneumonia, which consist of the following features: Ground Glass Opacities (GGO), crazy paving pattern, and consolidations with peripheral and basal predominance. These chest CT features are observed in peak stage [9-13] days after the onset of COVID-19 infection) [13, 14]. Even though India has been reporting a spike in COVID-19 cases, there is a scarcity of studies describing the chest CT findings in COVID19 patients in India $[15,16]$. Hence, uncertainty thrives concerning the chest CT features of COVID-19 in India. Given this context, we sought to investigate the chest CT features of COVID-19 pneumonia in the Indian population.

\section{Methods}

Study population

A single-center retrospective, observational study was conducted in 312 consecutive adult COVID-19 patients from 1 January 2021 to 31 March 2021 at Shukla Diagnostic Center, Balaghat, Madhya Pradesh, India.

\section{Inclusion and exclusion}

Irrespective of gender and availability of RT-PCR report, patients (more than 18 years of age) with typical symptoms of COVID-19 such as fever, cough, fatigue, sore throat, and dyspnea were included. All the patients referred to our private radio diagnostic center were subjected to high-resolution computed tomography (HRCT) chest. Malignancy, immunodeficiency, pregnancy, and absence of all medical records were the exclusion criteria.

\section{Sample size}

A sample size calculation was computed using Raosoft software. (http://www.raosoft.com/samplesize.html?nosurvey) on the assumption of a prevalence of disease of $50 \%$, and marginal error of $5.62 \%$ with a population of 20,000 and confidence interval of $95 \%$. The minimum sample size required would be at least 300 .

\section{Clinical and laboratory data collection}

The following data were recorded using a predefined proforma; a) baseline demographics, b) clinical and laboratory characteristics and c) extent, pattern, and type of abnormal CT findings. RT-PCR was performed on nasopharyngeal swabs or aspiration, using RealStar ${ }^{\circledR}$ SARS-CoV-2 RT-PCR Kit (Altona Diagnostics) or Cobas ${ }^{\circledR}$ SARS-CoV-2 Test (Roche).

\section{Chest CT protocols}

Patients were subjected to chest CT after collection of a nasopharyngeal swab. Chest CT was done on an average of 5.8 days (range 3-9 days) after symptom onset. All the chest CT scans were acquired on a 32 slice multi-detector CT unit (Siemens Somatom Go Now) without contrast medium injection. The scanning parameters were as follows: tube voltage of $110 \mathrm{kVp}$, tube current of $90-130 \mathrm{mAs}$, and collimation of $16 \times 0.6$, and a beam pitch of 1.5 . The CT images were obtained in a single inspiratory breath-hold. Using a high- frequency reconstruction algorithm, images were reconstructed using a reconstruction increment of $0.7-1 \mathrm{~mm}$ thick slices. All the images were viewed at lung (window width: 1200-1500 $\mathrm{HU}$; window center: 500-600HU) and mediastinal (window width: 300-400HU; window center: $40 \mathrm{HU}$ ) window settings. Decontamination of the CT suite was done using surface disinfectants (70\% ethanol or $0.1 \%$ sodium hypochlorite)..

\section{Image interpretation}

All CT images presented in the current study belong to our center (Shukla Diagnostic Center, Balaghat, Madhya Pradesh, India). CT images were analyzed on an Osirix MD workstation (Apple Mac). All chest CT images, including presence or absence of pulmonary opacities, location, type of opacities, and extent, were independently examined by two primary interpreting radiologists, who were not aware of any clinical or laboratory findings or patient outcomes. A third senior radiologist resolved any disagreement between two primary interpreting radiologists with discussion. The lung lesions were categorized into 1) right lung left lung, or bilateral, and 2) peripheral predominant (defined as outer one-third of the lung), central predominant (defined as the inner two-third of the lung tissue), or diffuse. The number of lobes involved was recorded. The lung abnormalities were also classified into anterior and posterior locations. The lung tissue anterior to a line drawn midway on axial CT and the portion behind it was defined as anterior and posterior, respectively. According to the Fleischner Society Glossary [17] for thoracic imaging, Lung lesions were enumerated for each patient. A semi-quantitative CT score was used to quantify the extent of pulmonary abnormalities by visually enumerating the percentage of the total lung involvement.

\section{Statistical analysis}

Discrete variables are expressed as frequencies, while continuous variables are expressed as mean $\pm \mathrm{SD}$. The agreement between two interpreting radiologists for CT findings was evaluated with the Kappa method. According to Landis and Koch, an inter-observer agreement was considered poor for a kappa value of 0.01-0.20, slight for 0.21-0.40, fair for 0.41-0.60, moderate for 0.61-0.80, and substantial for 0.81-1.0. There was almost a perfect agreement (Cohen's Kappa of 0.91) in reading CT images between the two primary radiologists. All analyses were performed using the Statistical Package for Social Sciences, version15 (SPSS, Chicago, IL, USA).

\section{Results}

Sociodemographic factors

Three hundred and twelve patients with a mean age of 43.18 ( \pm 8.27 years) were enrolled in the study. Most of the examined patients were males $(65.4 \%)$. Of the 312 patients, $228(73.1 \%)$ patients had a history of contact with RT-PCR confirmed COVID-19 or travel to a high-risk area within or outside the country. In our study population, males (65.4\%) were predominately infected with COVID-19. Cough was the most frequent $(48.1 \%)$ clinical presentation, followed by fever (47.1\%). The mean WBC count was 6.53( \pm 1.57$)$, whereasincreased lymphocyte and CRP count were found in 69 $(22.1 \%)$ and $270(86.5 \%)$ patients, respectively (Table 1$)$ 
Table 1 Demographics, clinical characteristics, and laboratory data for COVID-19 infected patients ( $\mathrm{N}=312$ )

\begin{tabular}{|l|l|}
\hline Characteristics & $\mathbf{N}(\%)$ \\
\hline Mean age, years & $43.18 \pm 8.27$ \\
\hline $\begin{array}{l}\text { History of contact with a COVID-19 } \\
\text { patient or travel to a high-risk zone }\end{array}$ & $204(65.4)$ \\
\hline Comorbidities & $228(73.1)$ \\
\hline Hypertension & $51(16.3)$ \\
\hline Diabetes mellitus & $24(7.7)$ \\
\hline Chronic liver disease & $18(5.8)$ \\
\hline Rheumatoid arthritis & $21(6.7)$ \\
\hline Clinical presentation & $147(47.1)$ \\
\hline Fever & $150(48.1)$ \\
\hline Cough & $51(16.3)$ \\
\hline Sore throat & $117(37.5)$ \\
\hline Dyspnea & $117(37.5)$ \\
\hline Malaise/Fatigue & $51(16.3)$ \\
\hline Increased respiratory rate $(>30 / m i n)$ & $57(18.3)$ \\
\hline Reduced oxygen saturation (<90\%) & \\
\hline Laboratory parameter & $6.53 \pm 1.57$ \\
\hline $\begin{array}{l}\text { Mean WBC count (normal value 4-11 } \\
\text { 109/L) }\end{array}$ & $243(77.9)$ \\
\hline $\begin{array}{l}\text { Lymphocyte count (normal value 1.1-3 } \\
\text { 109/L) }\end{array}$ & $270(86.5)$ \\
\hline Increased & \\
\hline Decreased & $69(22.1)$ \\
\hline Increased CRP (normal value < 10 mg/L) & \\
\hline
\end{tabular}

COVID-19, coronavirus disease; CRP: C- reactive protein; WBC, White blood cells, WBC: white blood cells

\section{Distribution of lung findings on chest CT}

A perfect agreement (Cohen's Kappa of 0.91) was observed in reading CT images between the two primary interpreting radiologists. Regarding the distribution of lung findings on chest CT examination, lung parenchymal abnormalities were reported in $294(94.2 \%)$ and normal chest CT in 18 (5.8\%) COVID-19 patients. The laterality of lung involvement was recorded in $294(94.2 \%)$ patients, and bilateral lung involvement $(45.6 \%)$ was the commonest among the patients with abnormal CT findings. The involvement of multiple lobes was the most common in patients with lung parenchymal abnormalities. Of the 312 patients, $126(42.9 \%)$ patients had involvement of five lobes. Concerning axial distribution, peripheral distribution was the most frequent axial location of opacity in $288(92.3 \%)$ patients (Figure 1), whereas none of the patients showed purely central distribution. Likewise, posterior distribution was found in $252(80.8 \%)$ patients in terms of anterior-posterior distribution (Table 2).

\section{The type of lung opacities}

The type of lung opacities and additional findings on CT are summarized in Table 3. GGO was the dominant abnormality observed in $270(91.8 \%)$ patients. Pure GGO was seen in $108(36.7 \%)$ patients. GGO with interlobular septal thickening and intralobular lines producing crazy-paving pattern was reported in 117(39.8\%) patients, while GGO mixed with consolidation was observed in 153(52.0\%) patients (Figure 2, 3, and 4).
Table 2 Distribution of lung findings on chest CT $(\mathrm{n}=312)$

\begin{tabular}{|l|l|}
\hline Lung parenchymal abnormalities on CT & N (\%) \\
\hline Normal CT & $18 / 312(5.8)$ \\
\hline Abnormal CT & $294 / 312(94.2)$ \\
\hline Laterality of lung involvement & \\
\hline Bilateral & $134 / 294(45.6)$ \\
\hline Right lung & $84 / 294(28.6)$ \\
\hline Left lung & $76 / 294(25.8)$ \\
\hline Right upper lobe & \\
\hline Right middle lobe & $126 / 312(40.4)$ \\
\hline Right lower lobe & $72 / 312(23.1)$ \\
\hline Left upper lobe & $108 / 312(34.6)$ \\
\hline Left lower lobe & $132 / 312(42.3)$ \\
\hline Number of lobes involved & $102 / 312(32.7)$ \\
\hline Five lobes & \\
\hline Four lobes & $126(42.9)$ \\
\hline Three lobes & $54(18.4)$ \\
\hline Two lobes & $46(15.6)$ \\
\hline One lobe & $32(10.9)$ \\
\hline Axial location of the opacity & $36(12.2)$ \\
\hline Central (inner 2/3rd of the lung) & \\
\hline Peripheral (outer 1/3rd of the lung) & 0 \\
\hline Central and Peripheral & $288(92.3)$ \\
\hline Antero-posterior location & $207(66.3)$ \\
\hline Anterior & \\
\hline Posterior & 0 \\
\hline Anterior and posterior & $252(80.8)$ \\
\hline & $108(34.6)$ \\
\hline congent & \\
\hline
\end{tabular}

CT: computed tomography

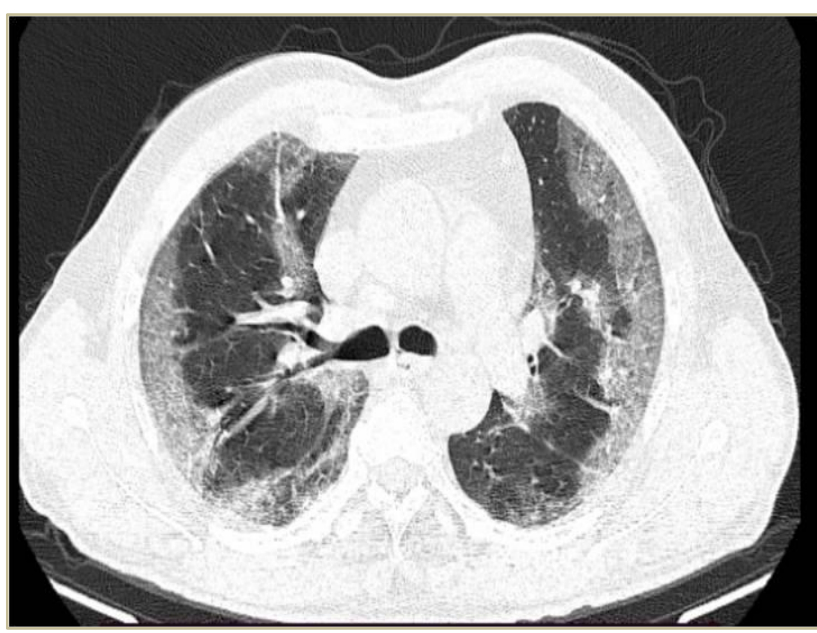

Figure 1: HRCT lung showing extensively peripheral subpleural involvement of lungs on both sides with thickening of interlobular interstitium within groundglass opacity in both lungs

Reticulation was observed in 126 (42.9\%) patients. A relatively smaller number of cases showed subpleural curvilinear lines in $45(15.3 \%)$ patients (Figure 5), air bronchogram sign in $90(30.6 \%)$ patients (Figure 6), halo sign in $15(3.1 \%)$ patients (Figure 7a), atoll or reverse halo sign in 45 (15.3\%) patients (Figure 7b), perilesional /intralesional vessel enlargement in $192(65.3 \%)$, and air bubble sign in $18(6.1 \%)$ patients. Fortyfive patients (15.3\%) showed bronchial wall thickening, 
whereas eighteen patients $(6.1 \%)$ showed bronchodilatation. Vasculopathy was found in $45(15.3 \%)$ patients (Figure 8). Spontaneous pneumomediastinum and spontaneous pneumothorax (Figure 9) was found in $18(6.1 \%)$ patients and $15(5.1 \%)$ patients, respectively. CT findings revealed that none of the patients showed pure consolidation, cavitation, pleural effusion, pericardial effusion, and mediastinal lymphadenopathy. A higher number [126 (42.9\%)] of patients with sequelae of old healed tuberculosis were observed in CT findings of existing lung disease, followed by emphysema $18(6.1 \%)$ and fibrosis/interstitial lung disease $18(6.1 \%)$.

Table 3 Type of lung opacities and additional findings on CT $(\mathrm{N}=312)$

\begin{tabular}{|l|l|}
\hline Lung opacities & $\mathbf{N}(\boldsymbol{\%})$ \\
\hline GGO & $270(91.8)$ \\
\hline Pure GGO & $108(36.7)$ \\
\hline GGO with crazy paving pattern & $117(39.8)$ \\
\hline Pure consolidation & 0 \\
\hline Mixed pattern (GGO with consolidation) & $153(52)$ \\
\hline Sub pleural linear/curvilinear lines & $45(15.3)$ \\
\hline Nodules & $15(5.1)$ \\
\hline Reticulations & $126(42.9)$ \\
\hline Halo sign & $15(5.1)$ \\
\hline Reverse halo sign & $45(15.3)$ \\
\hline Cavitation & 0 \\
\hline Perilesional /Intralesional vessel enlargement & $192(65.3)$ \\
\hline Bronchial wall thickening & $45(15.3)$ \\
\hline Bronchial dilatation & $18(6.1)$ \\
\hline Air bronchogram sign & $90(30.6)$ \\
\hline Air bubble sign & $18(6.1)$ \\
\hline Additional findings & \\
\hline Pleural effusion & 0 \\
\hline Pericardial effusion & 0 \\
\hline Mediastinal lymphadenopathy & 0 \\
\hline Vasculopathy & $45(15.3)$ \\
\hline Spontaneous pneumomediastinum & $18(6.1)$ \\
\hline Spontaneous pneumothorax & $15(5.1)$ \\
\hline Findings of existing lung disease & \\
\hline Emphysema & $18(6.1)$ \\
\hline Sequelae of old healed tuberculosis & $126(42.9)$ \\
\hline Fibrosis/ Interstitial Lung Disease & $18(6.1)$ \\
\hline
\end{tabular}

GGO: ground-glass opacities

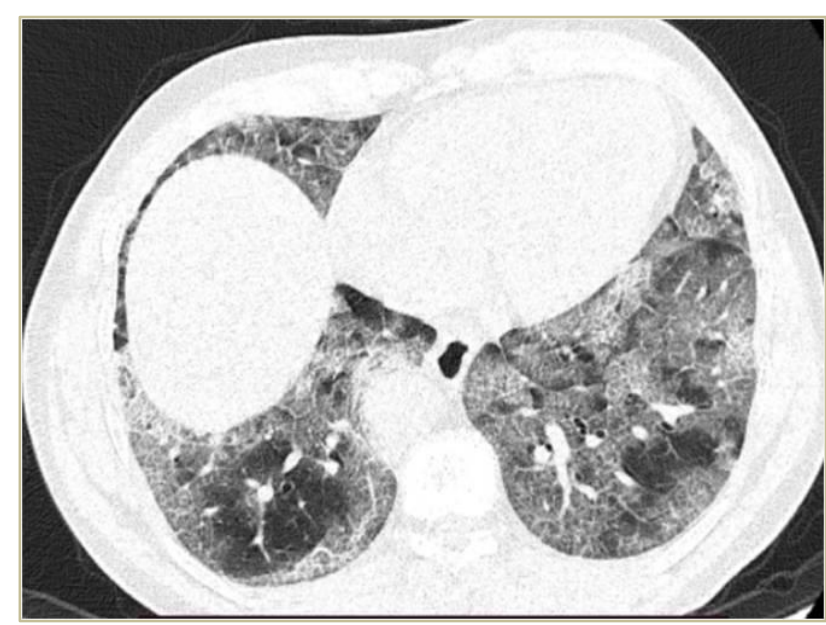

Figure 2: Crazy paving appearance: Image showing ground-glass opacity with thickening of the interlobular interstitium.

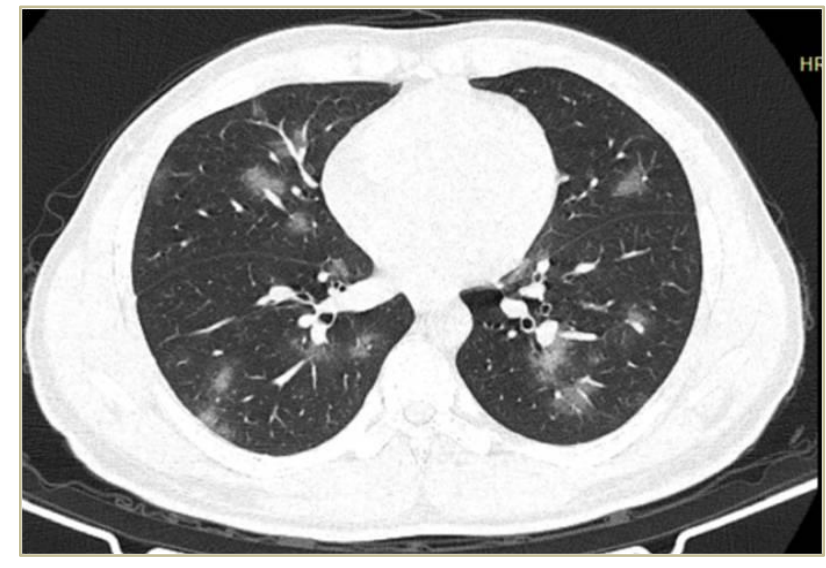

Figure 3: CT image of patients having symptoms of cough and fever showing early stages o COVID-19 infection in the form of multifocal peripheral subpleural and perivascular opacities with ground-glass attenuation in both lung

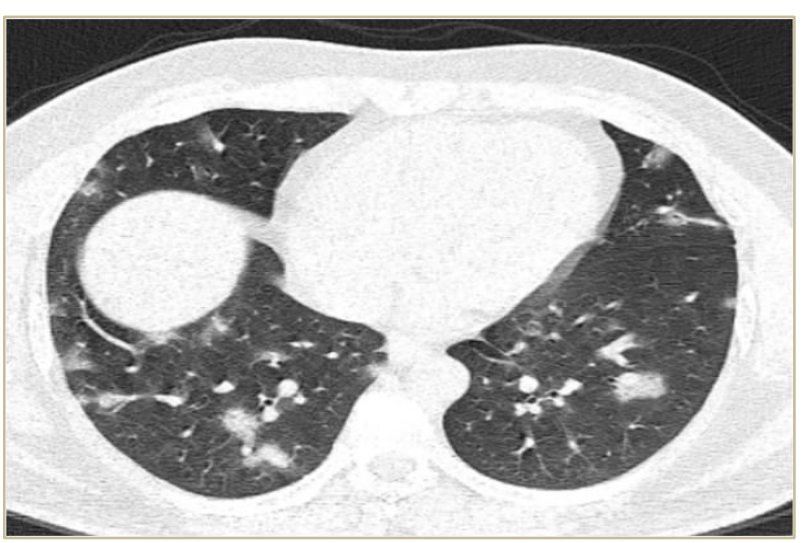

Figure 4: HRCT showing evolving nodular areas of consolidation in the subpleural and bronchogenic location in both lungs

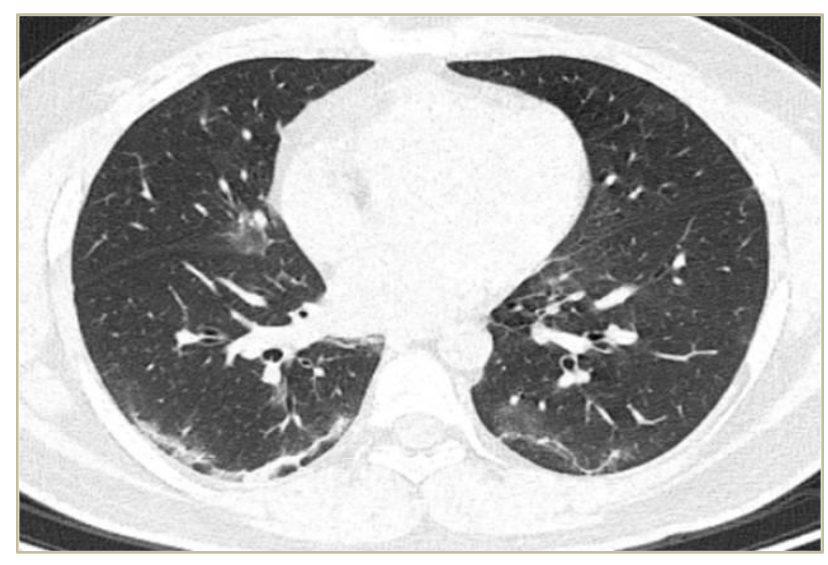

Figure 5: Image showing subpleural curvilinear areas of opacity in the right lower lobe

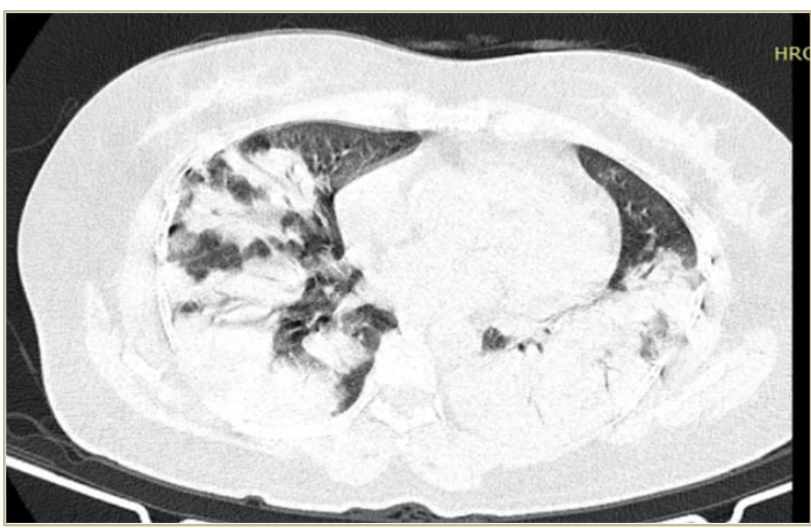

Figure 6: HRCT thorax of a patient with severe COVID Image showing diffuse ground-glass opacity intermixed with areas of consolidation in both lungs involving both subpleural and bronchogenic region with air bronchogram and dark bronchus sign 

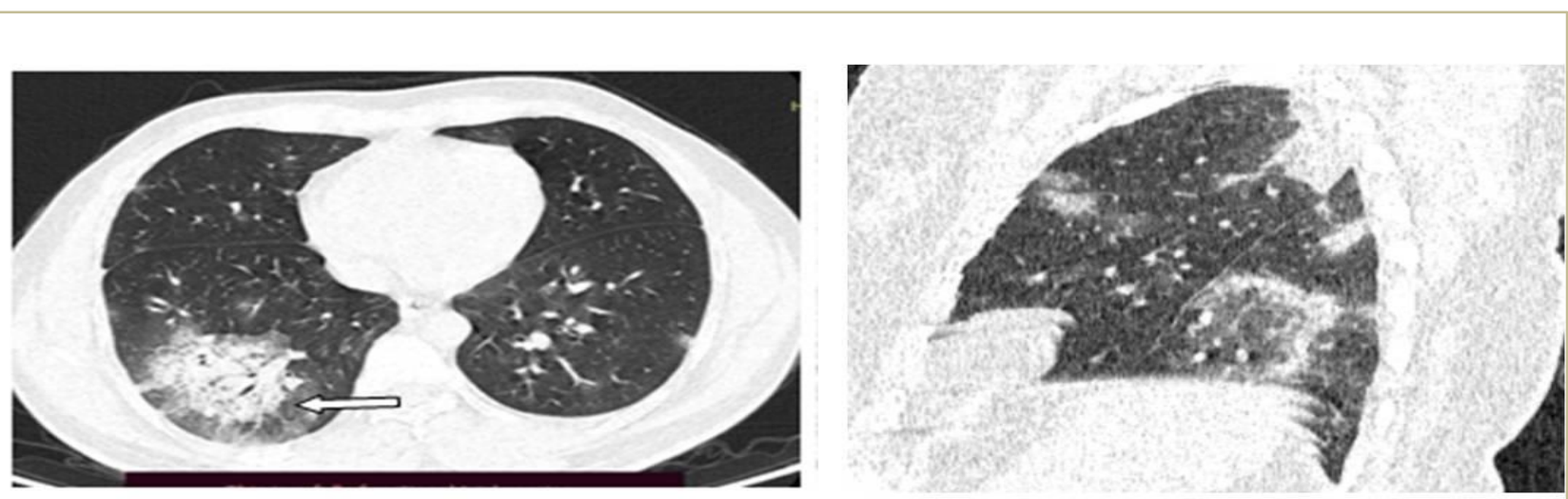

Figure 7: COVID -19 pneumonia simulating an organizing pneumonia pattern with the following signs: a) Halo sign: Image shows consolidation surrounded by groundglass opacity in the right lower lobe. Arrow indicated the ground glass opacity surrounding the consolidation, and b) Reverse Halo sign: Image shows the crescent of consolidation surrounding ground-glass opacity in the lower lobe of the left lung.
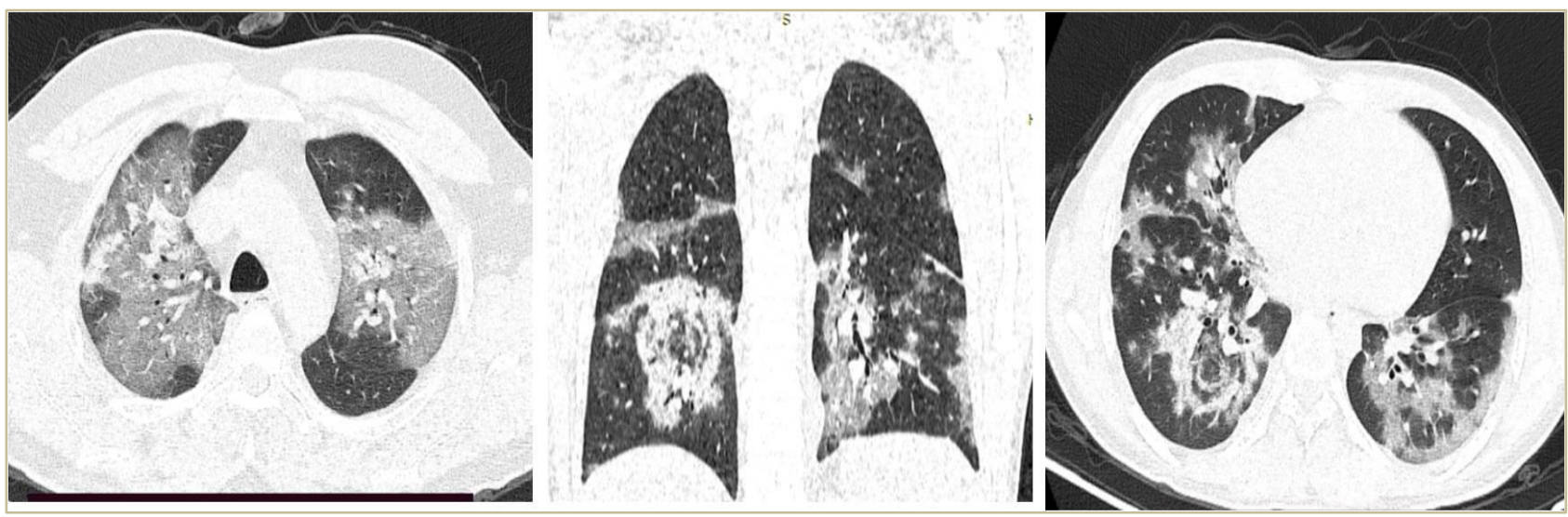

Figure 8: HRCT chest showing diffuse ground-glass opacity in upper lobes of both lungs with dilated subsegmental vessels within s/o vasculopathy
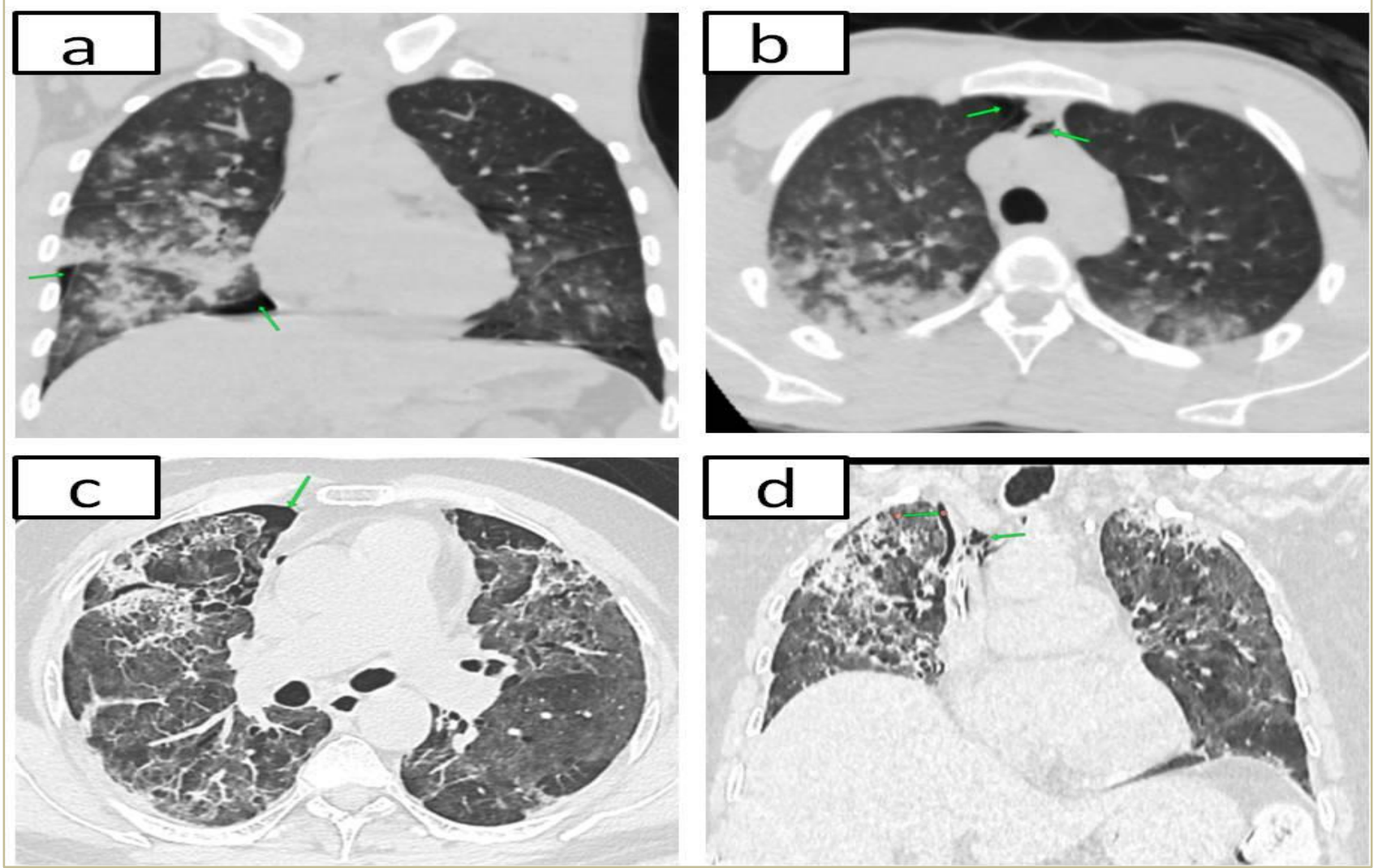

Figure 9: Coronal reformatted Image showing changes of covid pneumonitis in bilateral lung parenchyma with right-sided small pneumothorax (b) axial HRCT scan of the chest showing right-sided small pneumothorax with mild pneumomediastinum and changes of COVID pneumonitis in both lungs (c) axial HRCT scan of thorax showing mild right-sided pneumothorax with changes of COVID pneumonitis in both lungs (d) HRCT coronal reformatted Image showing mild right-sided pneumothorax with minimal pneumomediastinum with changes of COVID pneumonitis 


\section{Discussion}

Chest CT is a feasible modality to assess the severity of COVID-19 by evaluating lung pathology in COVID-19 patients [10]. The majority of the COVID-19 patients are asymptomatic during the early phase of the disease. CT scanning can identify the patients with minimal symptoms and those who were asymptomatic at admission. Positive chest CTs are found in asymptomatic patients, whereas, in contrast, negative chest CTs are found in symptomatic patients during the early phase of the disease $[13,15,16]$. In the present study, no lung parenchymal abnormalities were observed in the majority of the RT-PCR confirmed COVID-19 patients. Controversially, the higher percentage $(66.3 \%)$ of negative $\mathrm{CT}$ in the present study refute from previous results who have found lung parenchymal abnormalities in $71.8 \%$ [17], 96.6\% [18], 100\% [19], 88.7\% [20], $89.7 \%$ [21], 57.8\% [22], and $65.3 \%$ [13] RT-PCR confirmed COVID-19 patients. The discrepancy of the low incidence of CT findings in the present study with the previous studies may be due to the following reasons: 1) a Low number of comorbidities was found in the population. 2) The study was performed in all consecutive symptomatic RT-PCR confirmed COVID-19 patients, irrespective of the severity of symptoms; and 3) There is a high proportion of young patients (43.18 \pm 8.27 years) in our study. Pulmonary parenchymal abnormalities in crazy paving patterns and coalescing consolidations are associated with poor clinical outcomes. Pathologically, it may increase alveolar damage in the COVID-19 patients. The present study revealed the predominance of GGOs with posterior and peripheral predilection with a bilateral and multilobar distribution of pulmonary opacities among the patients with lung parenchymal abnormalities on chest CT. GGO was the dominant abnormality found in 45 (44\%) patients, in which pure GGO (11.5\%), GGO with superimposed crazy paving pattern $(12.5 \%)$, or GGO admixed with consolidation $(16.3 \%)$ were noted. The findings are consistent with the previous studies [22, 24]. The bilateral and mixed involvement of posterior and peripheral may be the lurking enemy behind the poor clinical outcomes in COVID-19 patients. The presence of vascular enlargement may be beneficial to differentiate COVID-19 pneumonia from nonCOVID-19 pneumonia, as vascular enlargement arises due to the vasodilatory effect of proinflammatory cytokines, small vessel pulmonary embolism, and infection-induced pulmonary vasculitis $[22,25]$. In concordance with the previous studies $[17,18,22]$, our study also found the segmental or subsegmental intra-lesional or peri-lesional pulmonary vessel enlargement. Certain limitations of this study are worthy of mention. Firstly, it was a single-center study with small sample size, so this result should be cautiously used to extrapolate CT predictive values, which may differ based on the disease prevalence, different populations, and periods. Secondly, we emphasized examining initial or baseline CT findings without follow-up and did not conduct follow-up to check for temporal modifications (progression, stability, or dissipation) in pulmonary abnormalities. Lastly, valuable information such as the presence or absence of underlying respiratory or cardiac comorbidities (or both) in the study population were not examined; thus, GGO, interlobular septal thickening, and pleural effusion could be due to the presence of underlying cardiac disease. Future controlled studies that segregate the direct pulmonary effects from the COVID-19 from those caused by the underlying medical diseases could prove beneficial for the future treatment of such patients.

\section{Limitation of study}

Certain limitations of this study are worthy of mention. Firstly, it was a single-center study with small sample size, so this result should be cautiously used to extrapolate CT predictive values, which may differ based on the disease prevalence, different populations, and periods. Secondly, we emphasized examining initial or baseline CT findings without follow-up and did not conduct follow-up to check for temporal modifications (progression, stability, or dissipation) in pulmonary abnormalities. Lastly, valuable information such as the presence or absence of underlying respiratory or cardiac comorbidities (or both) in the study population were not examined; thus, GGO, interlobular septal thickening, and pleural effusion could be due to the presence of underlying cardiac disease. Future controlled studies that segregate the direct pulmonary effects from the COVID-19 from those caused by the underlying medical diseases could prove beneficial for the future treatment of such patients.

\section{Conclusion}

This study can conclude that a higher incidence $(94.2 \%)$ of abnormal chest CTs in mildly symptomatic RT-PCR confirmed COVID-19 patients. Of note, CT findings revealed the manifestation of GGOs in a bilateral and multilobar distribution with posterior and peripheral predilection in COVID-19 pneumonia patients. However, this finding should be confirmed by more extensive studies.

\section{Abbreviation}

RT-PCR: Real-Time Polymerase Chain Reaction; CT: Computed Tomography; GGO: Ground Glass Opacity; Severe Acute Respiratory Syndrome Coronavirus 2: SARS-CoV-2, COVID-19: Coronavirus Disease

\section{Declaration}

Acknowledgment

The authors would like to express gratitude to all respondents who agreed to participate in this study.

Funding

The authors received no financial support for their research, authorship, and/or publication of this article.

Availability of data and materials

Data will be available by emailing akshat.shukla006@gmail.com.

\section{Authors' contributions}

Akshat Sanjay Shukla (ASS) and Sanjay Rajendraprasad Shukla (SRS) are the principal investigators of this manuscript. ASS is responsible for the study concept, design, writing, and approval of the final manuscript. SRS is responsible for reviewing and editing the manuscript in its final form. Feral Ravi Daruwala (FRD) assists in data analysis.

\section{Ethics approval and consent to participate}

We conducted the research following the Declaration of Helsinki. Ethical approval was obtained from Shukla Diagnostic Center, Balaghat, Madhya Pradesh - 480001 (Approval No: 2021/06) 
Consent for publication

Not applicable

Competing interest

The authors declare that they have no competing interests.

Open Access

This article is distributed under the terms of the Creative Commons $\begin{array}{llll}\text { Attribution } & 4.0 & \text { International License }\end{array}$ (http://creativecommons.org/licenses/by/4.0/), which permits unrestricted use, distribution, and reproduction in any medium, provided you give appropriate credit to the original author(s) and the source, provide a link to the Creative Commons license, and indicate if changes were made. The Creative Commons Public Domain Dedication waiver (http://creativecommons.org/publicdomain/zero/1.0/) applies to the data made available in this article unless otherwise stated.

\section{Author Details}

${ }^{1}$ Shukla Diagnostic Center, Balaghat, Madhya Pradesh, India.

Article Info

Received: 03 September 2021

Accepted: 31 October 2021

Published: 23 November 2021

\section{References}

1. Coronavirus disease 2019 (COVID-19) Situation [report] [cited May 5, 2021]. Available from: https:/www.who.int/docs/defaultsource/coronaviruse/situation-reports/20200121-sitrep-1-2019ncov.pdf?sfvrsn=20a99c10_4. [Accessed on 8 July 2021].

2. Ali Jadoo SA, Alhusseiny A, Yaseen S, Al-Samarrai M, Al-Rawi R, Al-Delaimy A, et al. Knowledge, attitude, and practice toward COVID-19 among Iraqi people: a web-based cross-sectional study. Journal of Ideas in Health. 2020;3(Special2):258-65. https://doi.org/10.47108/jidhealth.Vol3.IssSpecial\%202.59

3. World Health Organization. Coronavirus (COVID-19) dashboard [cited May 5, 2021]. Available from: https://covid19.who.int/.Accessed on 8 July 2021.

4. Yang Y, Yang M, Shen C, Wang F, Yuan J, Li J, et al. Laboratory diagnosis and monitoring the viral shedding of 2019-nCoV infections. MedRxiv. 2020.

5. Sverzellati N, Milone F, Balbi M. How imaging should properly be used in COVID-19 outbreak: an Italian experience. Diagn Interv Radiol. 2020;26(3):204-6. https://doi.org/10.5152/dir.2020.30320.

6. Simpson S, Kay FU, Abbara S, Bhalla S, Chung JH, Chung M, et al. Radiological Society of North America expert consensus document on reporting chest CT findings related to COVID-19: endorsed by the society of thoracic Radiology, the American College of Radiology, and RSNA. Radiol Cardiothorac Imaging. 2020;25(2):e200152.

7. Fang Y, Zhang H, Xie J, Lin M, Ying L, Pang P, et al. Sensitivity of chest CT for COVID-19: comparison to RT-PCR. Radiology. 2020;296(2): E115-7. https://doi.org/10.1148/radiol.2020200432.

8. Salehi S, Abedi A, Balakrishnan S, Gholamrezanezhad A. Coronavirus disease 2019 (COVID-19): a systematic review of imaging findings in 919 patients. AJR Am J Roentgenol. 2020;215(1):87-93. https://doi.org/ 10.2214/AJR.20.23034.

9. Zhou Z, Guo D, Li C, Fang Z, Chen L, Yang R, et al. Coronavirus disease 2019: initial chest CT findings. Eur Radiol. 2020;30(8):4398-406. https://doi.org/10.1007/s00330-020-06816-
10. Phan T. Genetic diversity and evolution of SARS-CoV-2. Infect Genet Evol. 2020;81(81):104260. https://doi.org/10.1016/j.meegid.2020.104260.

11. Shen Z, Xiao Y, Kang L, Ma W, Shi L, Zhang L, et al. Genomic diversity of SARS-CoV-2 in coronavirus Disease 2019 patients. Clin Infect Dis. 2020;4(71):713-20.

12. Ai T, Yang Z, Hou H, Zhan C, Chen C, Lv W, et al. Correlation of chest CT and RT-PCR testing for coronavirus disease 2019 (COVID-19) in China: a report of 1014 cases. Radiology. 2020;296(2):E32-40. https://doi.org/10.1148/radiol.2020200642.

13. Bernheim A, Mei X, Huang M, Fayad ZA, Zhang N, Diao K, et al Chest CT findings in coronavirus disease-19 (COVID-19): relationship to duration of infection. Radiology. 2020;20 (295):200463

14. Zuo H. Contribution of CT features in the diagnosis of COVID19. Can Respir J. 2020;2020:1237418. https://doi.org/10.1155/2020/1237418.

15. Parry AH, Wani AH, Yaseen M, Dar KA, Choh NA, Khan NA, et al. Spectrum of chest computed tomographic (CT) findings in coronavirus disease-19 (COVID-19) patients in India. Eur J Radiol. 2020;129:109147. https://doi.org/10.1016/j.ejrad.2020.109147.

16. Parry AH, Wani AH, Shah NN, Yaseen M, Jehangir M. Chest CT Features of coronavirus disease-19 (COVID-19) Pneumonia: which Findings on Initial CT Can Predict an Adverse Short-Term Outcome? BJR. 2020;2(1):20200016. https://doi.org/10.1259/bjro.20200016.

17. Tabatabaei SMH, Talari H, Moghaddas F, Rajebi H. CT Features and short-term prognosis of COVID-19 pneumonia: A singlecenter study from Kashan, Iran. Radiol Cardiothorac Imaging. 2020;2(2):e200130. https://doi.org/10.1148/ryct.2020200130.

18. Inui S, Fujikawa A, Jitsu M, Kunishima N, Watanabe S, Suzuki $\mathrm{Y}$, et al. Chest CT findings in cases from the cruise ship diamond princess with coronavirus disease (COVID-19). Radiol Cardiothorac Imaging. 2020;2(2):e200110. https://doi.org/10.1148/ryct.2020200110.

19. Chen Y, Li L. SARS-CoV-2: virus dynamics and host response. Lancet Infect Dis. 2020;20(5):515-6. https://doi.org/10.1016/S1473-3099(20)30235-8

20. Li K, Fang Y, Li W, Pan C, Qin P, Zhong Y, et al. CT image visual, quantitative evaluation and clinical classification of coronavirus disease (COVID-19). Eur Radiol. 2020;30(8):440716. https://doi.org/10.1007/s00330-020-06817-6.

21. Caruso D, Zerunian M, Polici M, Pucciarelli F, Polidori T, Rucci $\mathrm{C}$, et al. Chest CT features of COVID-19 in Rome, Italy. Radiology. https://doi.org/10.1148/radiol.2020201237.

22. Yu M, Xu D, Lan L, Tu M, Liao R, Cai S, et al. Thin-section ches CT imaging of COVID-19 pneumonia: a comparison between patients with mild and severe disease. Radiol Cardiothorac Imaging. 2020;2(2):e200126. https://doi.org/10.1148/ryct.2020200126

23. Ai T, Yang Z, Hou H, Zhan C, Chen C, Lv W, et al. Correlation of chest CT and RT-PCR testing for coronavirus disease 2019 (COVID-19) in China: a report of 1014 cases. Radiology. 2020;296(2):E32-40. https://doi.org/10.1148/radiol.2020200642.

24. Bao C, Liu X, Zhang H, Li Y, Liu J. Coronavirus disease 2019 (COVID-19) CT findings: a systematic review and meta-analysis. J Am Coll Radiol. 2020;17(6):701-9. https://doi.org/10.1016/j.jacr.2020.03.006.

25. Salehi S, Abedi A, Balakrishnan S, Gholamrezanezhad A Coronavirus disease 2019 (COVID-19): a systematic review of imaging findings in 919 patients. AJR Am J Roentgenol. 2020;215(1):87-93. https://doi.org/10.2214/AJR.20.23034. 